$\xi=-1$

\title{
Software implementation of LTE-advanced using matlab Simulink
}

\author{
Wessam Mostafa $^{1 *}$, Eman Mohamed ${ }^{2}$, Abdelhalim Zekry ${ }^{3}$ \\ ${ }^{1}$ Instructor at Electronics and Communication department, Modern Academy for Engineering and Technology, Cairo, Egypt \\ ${ }^{2}$ Doctor and Lecturer at Electronics and Communication department, Modern Academy for Engineering and Technology, Cairo, Egypt \\ ${ }^{3}$ Professor Doctor at Electronics and Communication department, Ain Shams University, Cairo, Egypt \\ *Corresponding author E-mail: Wessam.Mostafa.A@hotmail.com
}

\begin{abstract}
Long Term Evolution Advanced (LTE-A) is the evolution of the LTE that developed by $3^{\text {rd }}$ Generation Partnership Project (3GPP).LTEA exceeded International Telecommunication Union (ITU) requirements for $4^{\text {th }}$ Generation (4G) known as International Mobile Telecommunications (IMT-Advanced). It is formally introduced in October 2009. This paper presents a study and an implementation of the LTE-A downlink physical layer based on 3GPP release 10 standards using Matlab simulink. In addition, it provides the LTE-A performance in terms of Bit Error Rate (BER) against Signal to Noise Ratio (SNR) for different modulation and channel coding schemes. Moreover, different scenarios of Carrier Aggregation (CA) are modeled and implemented. The Simulink model developed for the LTE-A transceiver can be translated into digital signal processor DSP code or VHDL on FPGA code.
\end{abstract}

Keywords: Carrier Aggregation (CA); Component Carrier (CC); Long Term Evolution (LTE); Long Term Evolution-Advanced (LTE-A); Orthogonal Frequency Division Multiplexing (OFDM).

\section{Introduction}

The results of the feasibility study for the LTE-A are published in 3GPP Technical Report 36.912 [1]. This technical report showed that the LTE-A met the IMT requirement. LTE-A deals with the higher data rate, which used over wide transmission bandwidth. The 3GPP standard publications of LTE-A provided that the peak data rate is 3 Gigabits per second (Gbps) in the downlink and 1.5 Gbps in the uplink over a scalable bandwidth ranges from $20 \mathrm{MHz}$ to $100 \mathrm{MHz} 1$ ]. LTE-A met IMT requirements by adding new techniques to LTE release 8.These techniques become a part of LTE-A Release 10. A briefly description of these techniques are provided in. The performance analysis of LTE-A is described in. This analysis included the LTE downlink physical layer block diagram and focused on the Orthogonal Frequency Division Multiplexing (OFDM). The results from this analysis achieved 74 Mbps in the downlink. In order to increase data rate compared to that achieved in [5], a non-continuous four Component Carriers (CCs) is implemented using Matlab software code in. This implementation achieved 1.6 Gbps downlink data rate over a bandwidth of $100 \mathrm{MHz}$.

The contribution of this paper is to implement a complete LTE-A downlink physical layer by using Matlab R2010a simulink software. In addition the turbo coder provides an enhancement over a convolutional coder in terms of the processing gain. This paper achieves high data rate over a transmission bandwidth of $100 \mathrm{MHz}$ by implementing five different CA scenarios. One of these scenarios is the aggregation of five Asymmetric CCs, which represents the maximum number of CCs in release 10. This Simulink imple- mentation can be considered as a first step for the software defined radio implementation of the LTE-A transceiver system

This paper arranged as follows: Section 2 introduces an overview of LTE-A. An implementation of LTE-A down link physical layer including OFDM system description and 3GPP LTE standard parameters are introduced in section 3.Section4, contains a discussion of the LTE-A CA including its types implementation. The simulation results are presented in section 5. Finally, section 6 concludes the paper.

\section{LTE-A overview}

LTE-A release-10 is the first $4 \mathrm{G}$ release, which enhanced the capability and performance of LTE by offering high spectrum efficiency, lower latency, higher data rate and improved mobility. It has features summarized in this paper, like Multi Input Multi Output transmission (MIMO), Coordinated Multi-Point transmission and reception (COMP) and Relaying. One of the most important LTE-A features described and implemented in this paper is the Carrier Aggregation CA.

\subsection{Carrier aggregation $\mathrm{CA}$}

CA is commercially started in Korea in 2013. It is one of the most important features introduced in LTE-A as it increases transmitted data rate by increasing transmission bandwidth. CA is used to combine two or more LTE CC for single user to support wider transmission bandwidth that is not supported in release 8 or 9 [1]. 


\subsection{Multi input multi output (MIMO)}

The multiple transmit and multiple receive antennas is another technique used to increase the capacity of the LTE system. LTE release 8 supports MIMO with a maximum $4 \times 4$ configuration in the downlink and $1 \times 2$ configurations in the uplink. LTE-A improves average data rate, cell edge performance and gain spectrum efficiency by extended MIMO scheme. LTE-A supports MIMO configuration up to $8 \times 8$ in the downlink and up to $4 \times 4$ in the uplink [1].

\subsection{Coordinated Multi-Point Transmission and Recep- tion (COMP)}

COMP is a technique that improved LTE-A system throughput, cell edge performance, and data rate. The frequency reused in each cell causes a capacity decrease due to other cell interference. COMP turns this interference into a useful signaling. This desired dynamic coordinated transmission includes joint transmission, from multiple geographically separate points and joint received signals at multiple separated points [1], [3], [13].

\subsection{Relaying}

Relaying is a technique that is introduced in LTE-A to enhance the performance of LTE in term of throughput and coverage. In the basic relay scheme, the User Equipment (UE) connects to the Relay Node (RN), while Relaying is a technique that is introduced in LTE-A to enhance the performance the relay connects to a donor cell of a donor enhanced Node B (eNB). RN can communicate with eNB in two ways: inband or outband. Inband communication uses the same band that eNB uses to communicate with UEs. In outband communication, different bands are used [4], [13].

\section{LTE-A downlink physical layer implemen- tation system model}

Downlink provides a communication from the eNB to UE. The function of LTE physical layer is to encode the binary digit into signals and to transmit and receive these signals across the communication media [5]. In this section, the model of the LTE-A downlink physical layer will be described and implemented. The function of each block and its parameter will be presented. The transmission structure of LTE-A has the same format as LTE. LTE has different transmission bandwidth of 1.4, 3, 5, 10, and 20MHz. All building blocks are implemented in this section for the bandwidth of $10 \mathrm{MHz}$. Fig.1 depicts the implemented block diagram of LTE downlink physical layer based on 3GPP specification standards. Table 1 shows the implemented system model parameters selected in this section.

Table 1: The Implemented System Model Parameters

\begin{tabular}{ll}
\hline Simulated input data & 1200 -bits \\
\hline Turbo coder rate & $1 / 3$ \\
Selected mapper order & 64 -QAM \\
Sub-carrier number & 600 \\
IFFT size & 1024 \\
OFDM symbols number & $1-$ Symbol \\
\hline
\end{tabular}




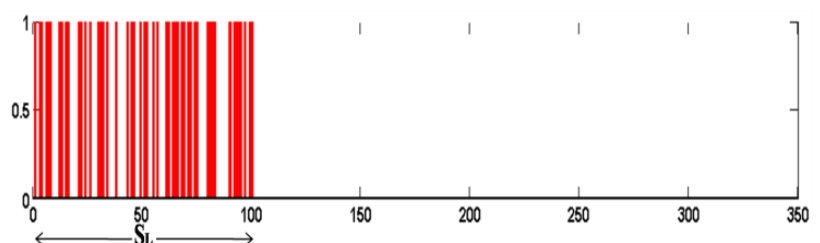

a. Turbo encoder input.

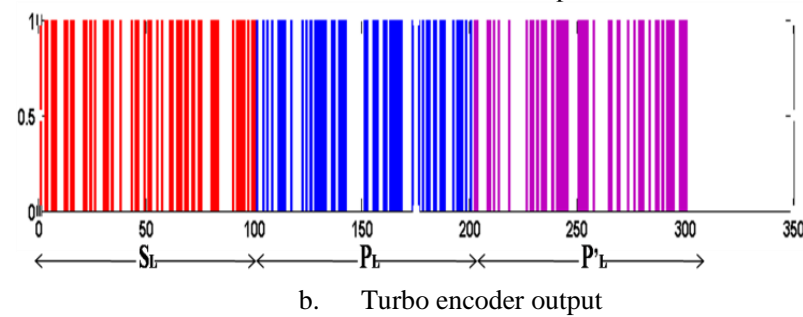

Fig. 3: a. The Input Bit Stream of Length 100 Bit which Applied to Turbo Encoder. b. The Output Bit Stream of Length 300 Bit.

\subsection{Rate match}

The turbo encoder output is fed to the rate match block. This block provides the desired output bit stream needed by the mapper block. By adding zero bits if needed [17].

\subsection{Mapper}

The bandwidth efficiency of communication systems is increased by modulating the coded bit stream into symbols. LTE downlink supports different mapping schemes like Quadrature Phase Shift Keying (QPSK), 16 Quadrature Amplitude Modulation (16-QAM) and 64 QAM with two, four and six bits per mapped symbol respectively. Higher modulation 256 QAM is also proposed. Fig.4 shows the constellation diagram of these mapper techniques.

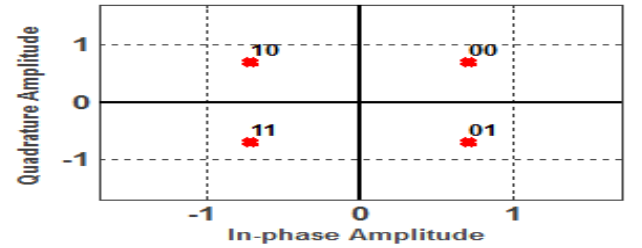

a. QPSK constellation diagram.

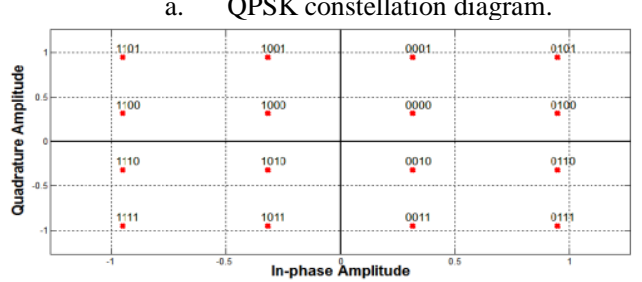

b. 16QAM constellation diagram.

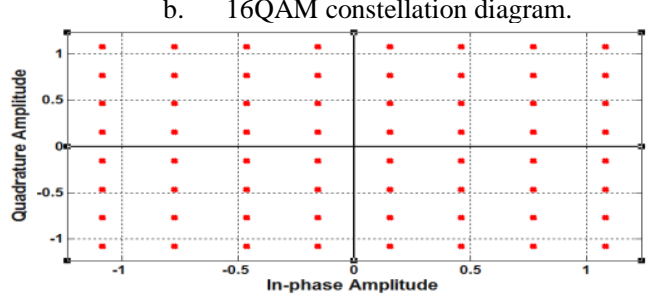

c. 64QAM constellation diagram

Fig. 4: The Constellation Diagram of Different Mapper Order Used in LTE A. QPSK B. 16-QAM C. 64-QAM
LTE allows eNB to choose modulation scheme according to the channel condition. As the mapper order increased, the transmission bandwidth efficiency is increased, at the price of increasing the BER for the same SNR.So 16-QAM or 64-QAM mapper order are used in case of good channel conditions and QPSK mapper order is used in case of poor channel coditions.

\subsection{OFDM symbol generator}

Orthogonal Frequency Division Multiplexing is the main technology of LTE and LTE-A. It is a multicarrier modulation technique, that is used to increase the transmission data rate and bandwidth efficiency by dividing the avaliable bandwidth into a number of overlapped sub-carriers. To avoid Inter-Carrier Interferance (ICI) problem, all sub-carriers are made orthogonal to each other [5], [19]. The simulink implementation of OFDM symbol generator is illustrated in fig.5.

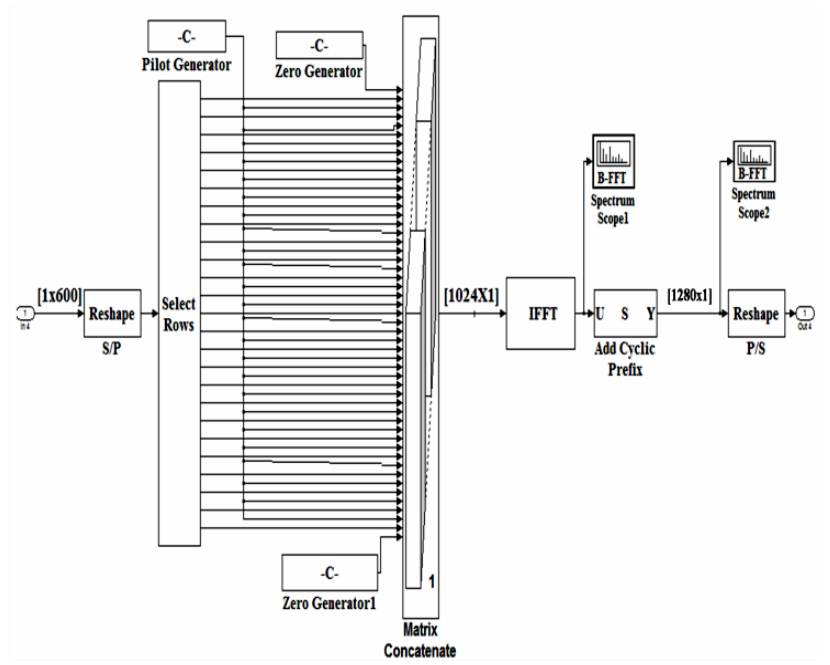

Fig. 5: The Simulink Implemented OFDM Symbol Generator.

In the implemented OFDM symbol generator, the "Reshape" block or serial to parallel converter is used to convert serial input symbols stream into parallel form. This form is suitable for Inverse Fast Fourier Transform (IFFT) requirement. The output from the reshape block is fed to "Select Rows" block. This block is used as a channel selector in order to distribute its input into a number of sub-channels. The IFFT block inputs are $\mathrm{N}_{\mathrm{FFT}}$ mapped symbols, which are consisting of ( 600 data +375 zeros +49 pilots). These inputs are combined using Matlab simulink block "Matrix concatenation". The output samples of IFFT are combined to construct a single OFDM symbol at time domain with length equal $\mathrm{N}_{\mathrm{FFT}} \mathrm{T}$, where $\mathrm{T}$ is the sampling period. FFT block is used on the receiver side to reconstruct the original mapped symbols [17].The "Add Cyclic Prefix (CP)"block is used to reduce Inter-Symbol Interference (ISI) and ICI by copying the last part of the OFDM symbol and inserting it at the beginning of the OFDM symbol as shown in fig.6. The useful symbol time is denoted by $\mathrm{T}_{\mathrm{u}}$ and $\mathrm{CP}$ time is denoted by $\mathrm{T}_{\mathrm{CP}}$. Therefore, the total OFDM symbol time after $\mathrm{CP}$ insertion is equal to $\left(\mathrm{T}_{\mathrm{u}}+\mathrm{T}_{\mathrm{CP}}\right)$ [19].

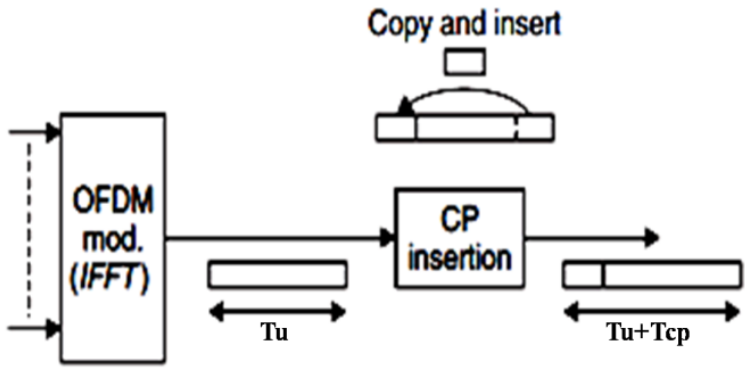

Fig. 6: CP Insertion [19]. 
"Spectrum Scope" is a Matlab simulink built in block, which is used to display output spectrum. Fig.7.a shows the Matlab simulink output symbol from IFFT in the frequency domain, before inserting CP for $10 \mathrm{MHz}$ bandwidth. Fig.7.b shows the IFFT output symbol after added long CP. one sees that the bandwidth of the signal has been increased by adding the cyclic prefix.

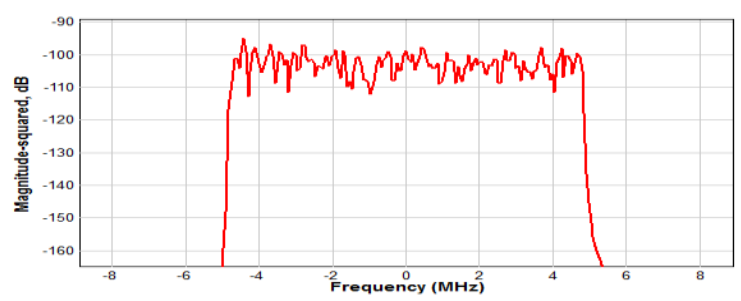

a. The input spectrum of "Add Cyclic Prefix" block.

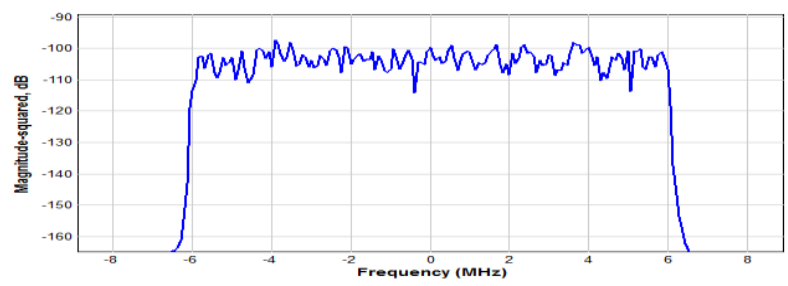

b. The Output Spectrum of "Add Cyclic Prefix" Block.

Fig. 7: a. CP Input Spectrum b. CP Output Spectrum.

\subsubsection{OFDM parameters selection for LTE downlink physical resources}

In OFDM system implementation, there are a number of basic parameters that must be considered, such as the number of data sub-carriers $\mathrm{N}_{\mathrm{sc}}$, CP length $\mathrm{N}_{\mathrm{cp}}$, FFT size $\mathrm{N}_{\mathrm{FFT}}$ and sampling frequency $\mathrm{F}_{\mathrm{S}}$ [19], [20].

In LTE downlink physical layer, OFDM sub-carrier spacing $\Delta \mathrm{F}$ is equal to $15 \mathrm{KHz}$. In Multicast/Broadcast over Single Frequency Network (MBSFN), LTE-A has reduced sub-carrier spacing to 7.5 $\mathrm{kHz}$ [19].

Resource Blocks RBs are another important parameter that must be considered in the LTE implementation. It is consisted of 12 consecutive sub-carriers in the frequency domain. Therefore, the resource block bandwidth is equal to $180 \mathrm{kHz}$. The total number of LTE downlink resource block $\left(\mathrm{N}_{\mathrm{RB}}\right)$ is ranging from 6 to more than 100 resource blocks.

All the parameters must be chosen to be compatible with each other. For example, if LTE transmission bandwidth is $10 \mathrm{MHz}$, the total number of resource blocks can be 50 resource blocks. Therefore, the total number of sub-carriers is equal to 600 sub-carriers. Then the suitable FFT size has been chosen as 1024 with sampling frequency is equal to $\Delta \mathrm{f} \times \mathrm{N}_{\mathrm{FFT}}=15 \mathrm{kHz} \times 1024=$ 15.36MHz. Table 3 presents the 3GPP LTE standard parameters for different transmission bandwidths.

Table 3: The 3gpp Lte Downlink ofdm Standard Parameters [19], [23]

\begin{tabular}{cccccc}
\hline Transmission BW (MHz) & 1.4 & 3 & 5 & 10 & 20 \\
\hline$\Delta \mathrm{f}(\mathrm{KHz})$ & 15 & 15 & 15 & 15 & 15 \\
$\mathrm{~N}_{\mathrm{RB}}$ & 6 & 15 & 25 & 50 & 100 \\
$\mathrm{~N}_{\mathrm{sc}}$ & 77 & 180 & 300 & 600 & 1200 \\
$\mathrm{~N}_{\mathrm{FFT}}$ & 128 & 256 & 512 & 1024 & 2048 \\
$\mathrm{~F}_{\mathrm{s}}, \mathrm{MHz}$ & 1.92 & 3.84 & 7.68 & 15.36 & 30.72 \\
Short $_{\mathrm{cp}}$ & 9 & 18 & 36 & 72 & 144 \\
Long N $_{\mathrm{cp}}$ & 32 & 64 & 128 & 256 & 512 \\
\hline
\end{tabular}

In the time domain, LTE frame duration is $10 \mathrm{~ms}$, each frame is divided into 10 sub-frames. Each sub-frame consists of two-time slots with time duration equal to $0.5 \mathrm{~ms}$. One time slot contains either 7 or 6 OFDM symbols, corresponding to short (normal) or long (extended) cyclic prefix, respectively. Fig.8 shows LTE generic frame structure. Each time slot is consisted of $1 \mathrm{RB}$. In the case of extended (long) cyclic prefix each resource block consist of 12 sub-carrier x 6 OFDM symbol $=72$ Resource Element RE. In the case of normal (short) cyclic prefix each resource block consist of 12 sub-carrier x 7 OFDM symbol = 84 RE. Resource Element (RE) is the smallest LTE downlink transmission time-frequency unit [12], [22], as depicted in fig.9.

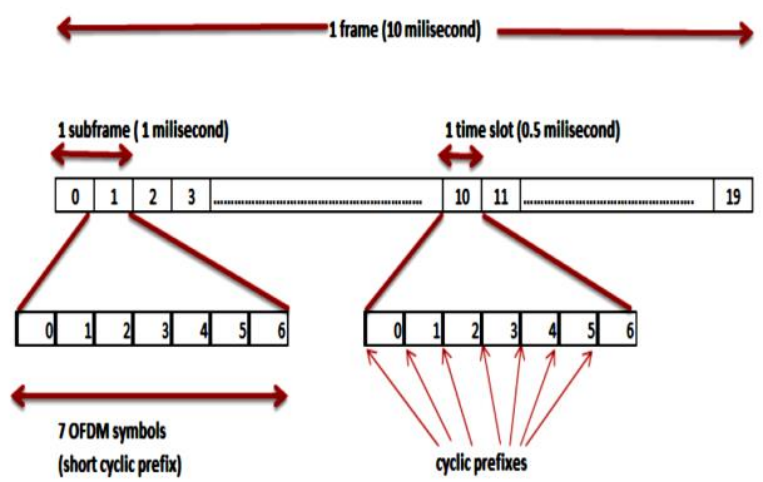

Fig. 8: LTE Generic Frame Structure [24].

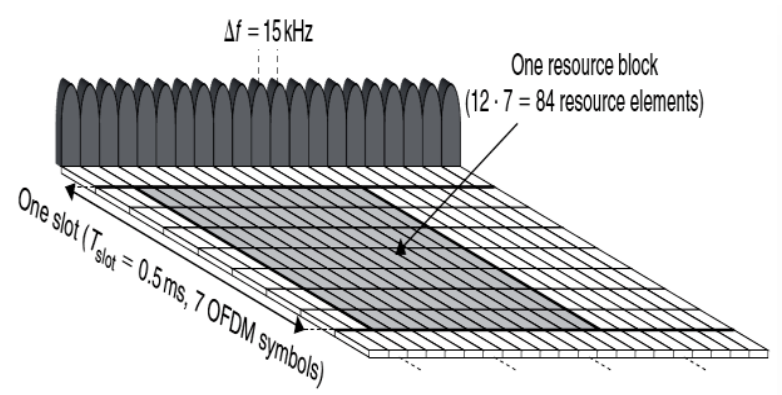

Fig. 9: LTE Downlink Resource Block for Normal Cyclic Prefix [22]

\section{LTE-A Carrier Aggregation CA.}

CA is one of the LTE-A new techniques introduced and defined in release 10. CA technique is used to aggregate two or more LTE carrier components CCs over the available spectrum; each CC has a bandwidth of $1.4,3,5,10,15$, or $20 \mathrm{MHz}$ to verify backward compatibility with release- 8 as shown in fig.10. This technique increases the peak data rate over wider transmission bandwidth.

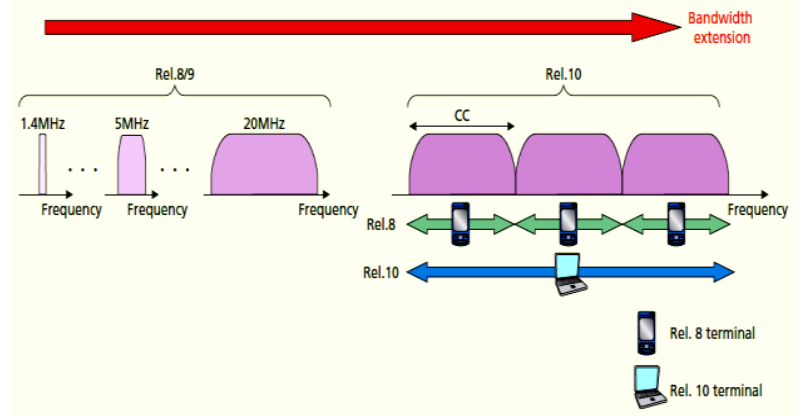

Fig. 10: CA Bandwidth Extension [26]. 
In 3GPP Release 10, the maximum number of CCs can be aggregated is five, to provide maximum aggregated bandwidth equals to $100 \mathrm{MHz}$. 3GPP Release-13 tries to increase the number of aggregated CCs to $32 \mathrm{CCs}$ [11].

\subsection{Carrier aggregation types}

There are three types of CA technique for LTE-A:

- Intra-band continuous carrier aggregation is the combination of adjacent $\mathrm{CC}$ in the same frequency band with spacing between center frequencies equal to multiple of 300 $\mathrm{KHz}$ that satisfy compatibility with LTE release 8, 9 and saving $15 \mathrm{KHz}$ sub-carrier spacing orthogonally [6], [11], as shown in fig. 11. a. It is easier to implement continuous CA than non-continuous $\mathrm{CA}$ implementation without making many changes in the physical layer. However, it is difficult to allocate $100 \mathrm{MHz}$ continuously, due to the spectrum allocation policies.

- Intra-band non-continuous carrier aggregation is the combination of non-adjacent $\mathrm{CC}$ over the same frequency band as shown in fig.11.b. Non-continuous CA is more complicated than the continuous type because it requires more advanced User Equipment (UE) transceiver .However, it solves the problem of continuous $100 \mathrm{MHz}$ allocation so that the noncontinuous CA implementation is more practical [6], [11], [27].

- Inter-band non-continuous carrier aggregation is the combination of the non-adjacent component carrier over different frequency bands as shown in fig.11.C. With this CA type, mobility robustness may be enhanced by profiteering different bands propagation characteristics [6], [11].

\section{Band A}

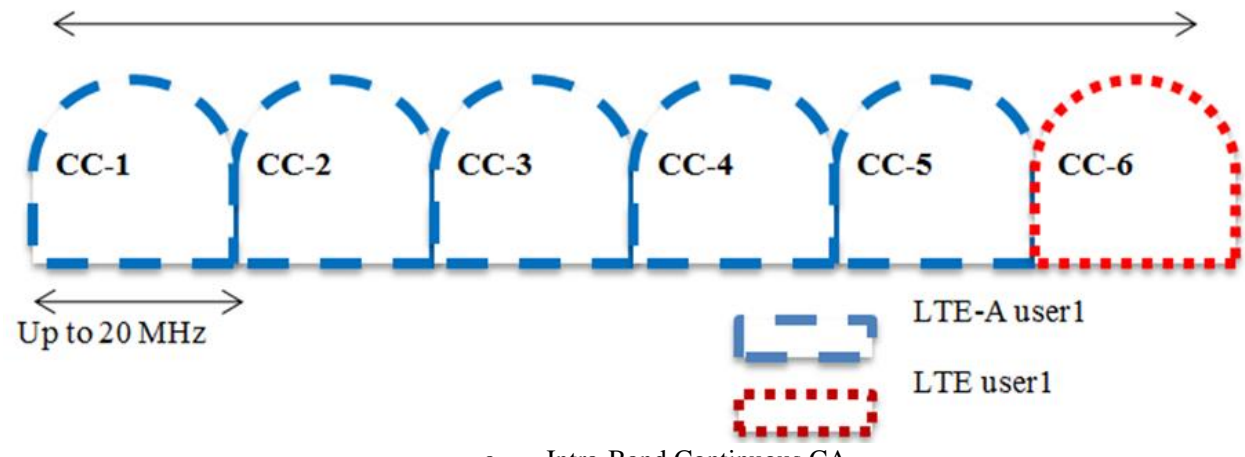

a. Intra-Band Continuous CA

Band A

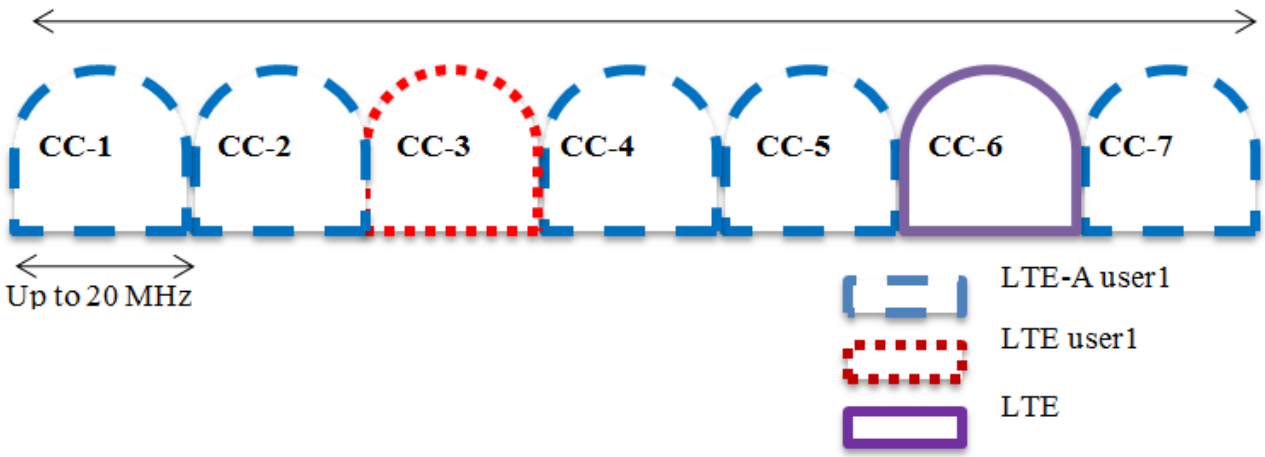

Band A

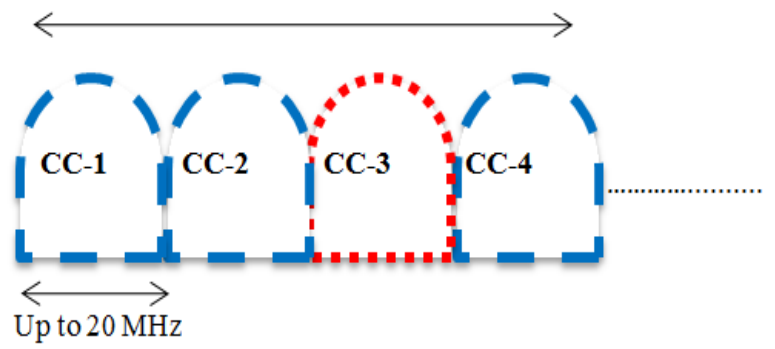

Band B

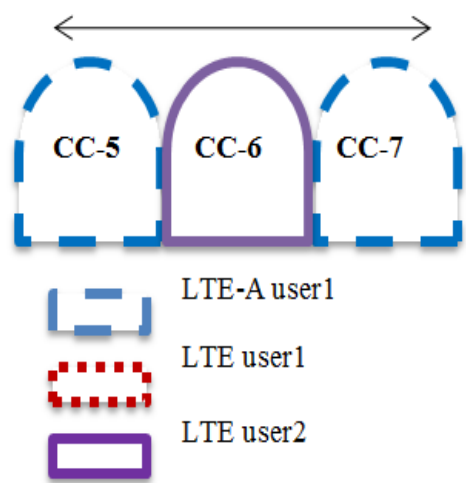

c. Inter-band non-continuous CA

Fig. 11: Types of CA (A) Intra-Band Continuous CA (B) Intra-Band Non-Continuous CA (C) Inter-Band CA [27] Carrier Aggregation Implementation 


\subsection{Carrier Aggregation implementation}

There are multi possible options for CA implementation. Fig.12 shows the first and simplest continuous intra-band CA implementation. This implementation consists of "Concatenation" block which combines and rearranges user frames, single IFFT, "Frequency Shift" block that makes frequency offset with center frequency $\left(\mathrm{F}_{\mathrm{C}}\right)$ and single Radio Frequency-Power Amplifier (RFPA). This implementation verifies the backward compatibility with LTE [6], [27], [30].

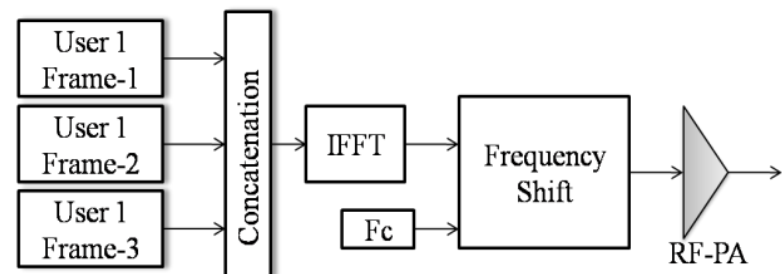

Fig. 12: The Implemented Block Diagram of Downlink Transmitter Continuous CA.

Continuous and non-continuous intra-band downlink transmitter Matlab simulink implementation is shown in fig.13. There are multiple of baseband LTE transmitters. Each of them is applied to frequency shift block. Then the output of all frequency shifts is combined using Matlab simulink block "Concatenation". Then the concatenation output is fed to signal radio frequency power amplifier RF- PA [6], [27].

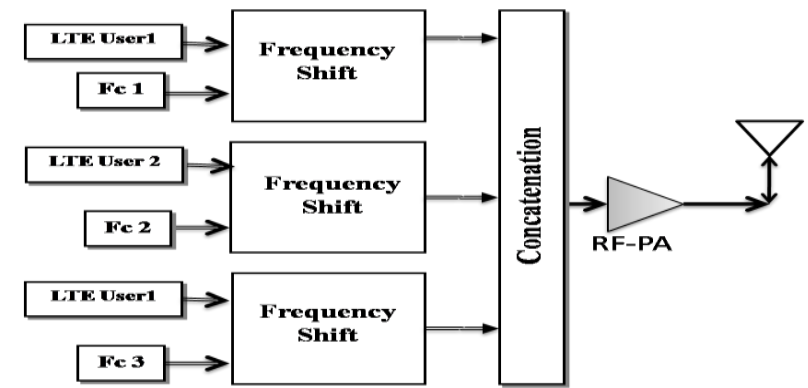

Fig. 13: The Simulink Implemented Block Diagram of Continuous and Non-Continuous Intra-Band CA Downlink Transmitter.

Fig.14 shows the Inter-band CA implementation. There is frequency up converter and PA for each component carrier before combining them. Note that, there are other possible implementations [27], [30].

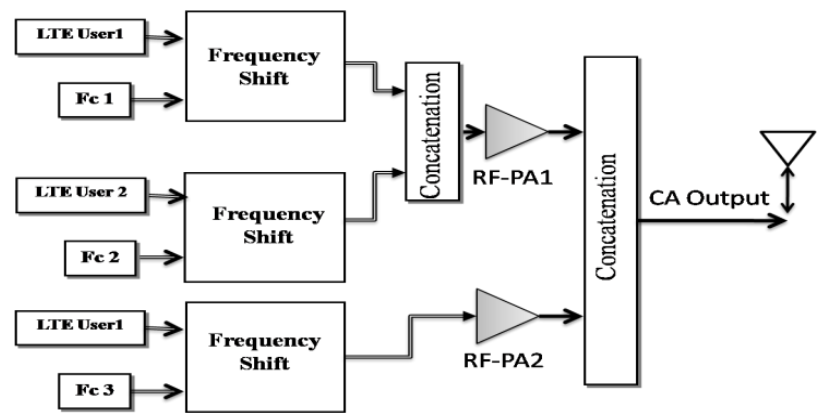

Fig. 14: The Simulink Implemented Block Diagram of Non-Continuous Inter-Band CA Downlink Transmitter.

\section{Simulation results}

In this section, we will introduce the results of the simulation of the modeled and implemented LTE-A with the parameters introduced in the previous sections. The implementation of LTE-A is executed using Matlab Simulink R2010a based on 3GPP standards parameters.

\section{The BER performance of LTE-A}

The first performance parameter of the implemented LTE-A system is the bit error rate as a function of the energy per bit to noise density ratio $\left(\mathrm{E}_{\mathrm{b}} / \mathrm{N}_{0}\right)$. Fig. 15 shows the tradeoff between the BER and the energy bit/Noise energy $\left(\mathrm{E}_{\mathrm{b}} / \mathrm{N}_{0}\right)$ at different mapper order for LTE-A turbo coder of $10 \mathrm{MHz}$ band, and the theoretical convolutional coder. This figure demonstrates that BER decreases by increasing $\mathrm{E}_{\mathrm{b}} / \mathrm{N}_{0}$. On the other hand, lower modulation order gives better BER performance for the same $E_{b} / N_{0}$. Also, Turbo coder provides an enhancement over convolutional coder in terms of the processing gain as apparent from Table 4.

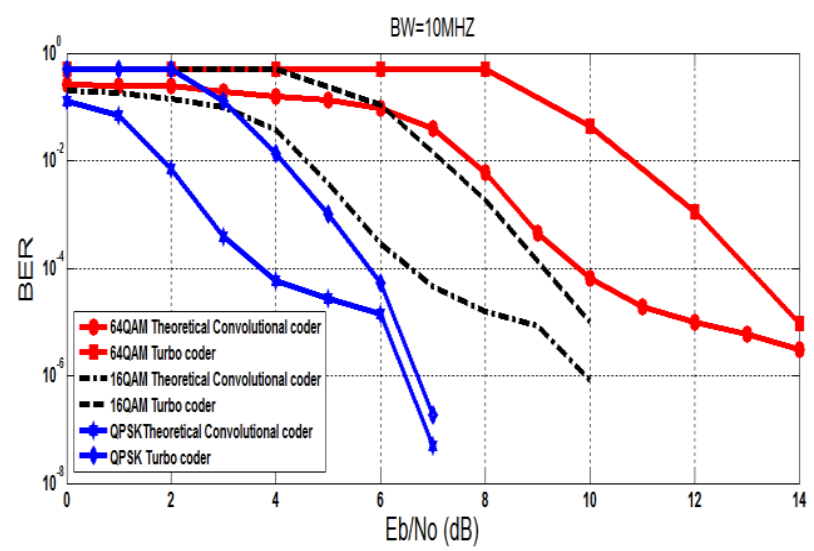

Fig. 15: BER Performance for LTE-A Turbo Coder and Theoretical Convolutional Coder at Different Mapper Order.

Table 4: Turbo Coder Performance Enhancement for BER $=10^{-5}$

\begin{tabular}{cccc}
$\begin{array}{c}\text { Mapper } \\
\text { order }\end{array}$ & $\begin{array}{c}\text { Turbo coder } \\
\mathrm{E}_{\mathrm{b}} / \mathrm{N}_{0}\end{array}$ & $\begin{array}{c}\text { Convolutional coder } \\
\mathrm{E}_{\mathrm{b}} / \mathrm{N}_{0}\end{array}$ & $\begin{array}{c}\text { Saving } \\
\mathrm{E}_{\mathrm{b}} / \mathrm{N}_{0}\end{array}$ \\
\hline 64-QAM & $9.8 \mathrm{~dB}$ & $13 \mathrm{~dB}$ & $3.8 \mathrm{~dB}$ \\
16-QAM & $6.8 \mathrm{~dB}$ & $9 \mathrm{~dB}$ & $2.2 \mathrm{~dB}$ \\
QPSK & $3.8 \mathrm{~dB}$ & $4.8 \mathrm{~dB}$ & $1 \mathrm{~dB}$ \\
\hline
\end{tabular}

\subsection{Implementation of different CA scenarios in LTE-} A

The simulation results of different implemented CA Scenarios using Matlab simulink are presented in this subsection.

\subsubsection{Scenario A}

Scenario A presents two CCs symmetric non-continuous $30 \mathrm{MHz}$ $\mathrm{CA}$ as shown in fig.16. Each CC has $10 \mathrm{MHz}$ bandwidth which is denoted as symmetric $\mathrm{CA}$. The first $\mathrm{CC}$ has a center frequency at $15 \mathrm{MHz}$ and the second $\mathrm{CC}$ has a center frequency at $36 \mathrm{MHz}$. So, the center frequency spacing is $21 \mathrm{MHz}$ which realizes the required multiple of $300 \mathrm{KHz}$

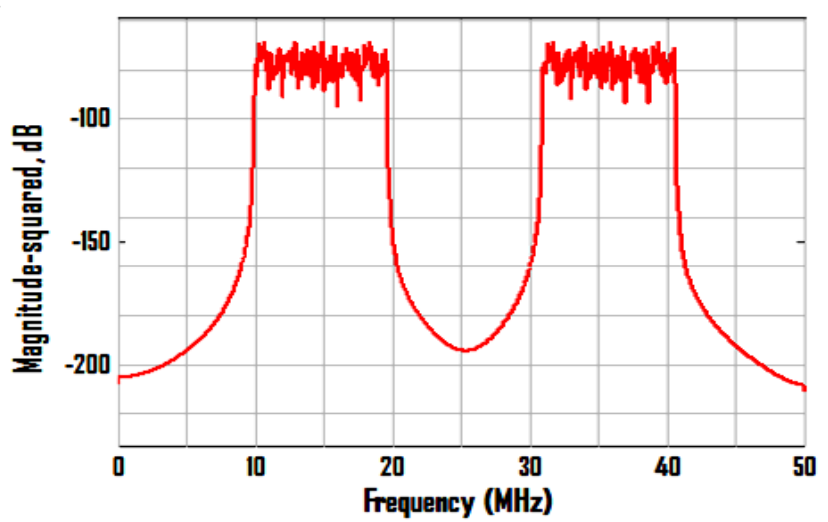

Fig. 16: Two CCs Non-Continuous Symmetric CA $30 \mathrm{MHz}$ 


\subsubsection{Scenario B}

Scenario B presents Two CCs Asymmetric non-continuous 60 $\mathrm{MHz} \mathrm{CA}$ as shown in fig.17. The first $\mathrm{CC}$ has $10 \mathrm{MHz}$ bandwidth with center frequency at $15 \mathrm{MHz}$. While as the second $\mathrm{CC}$ has 20 $\mathrm{MHz}$ bandwidth with center frequency at $60 \mathrm{MHz}$. So, the center frequency spacing is $45 \mathrm{MHz}$. This implemented CA is denoted as Asymmetric CA.

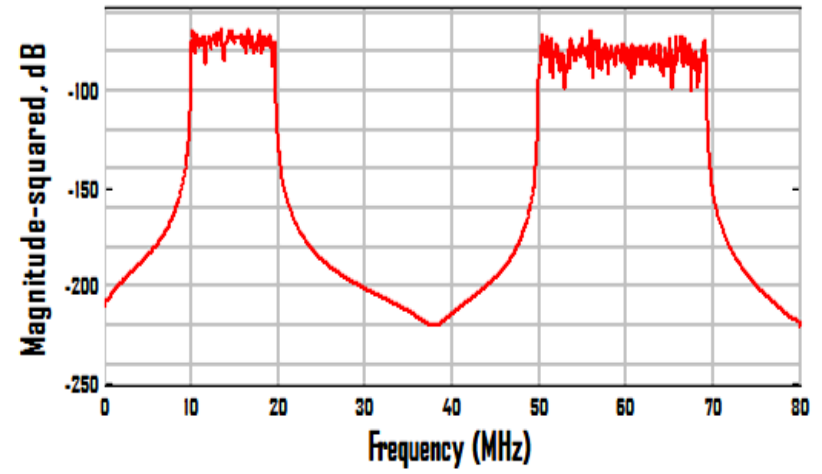

Fig. 17: Two CCs Non-Continuous Asymmetric CA 60 Mhz Bandwidth.

\subsubsection{Scenario $C$}

In order to increase the throughput of the system, scenario $\mathrm{C}$ presents Asymmetric intra-band non-continuous $60 \mathrm{MHz} \mathrm{CA}$ by using three CCs as shown in fig.18. The first two CCs have $10 \mathrm{MHz}$ bandwidths with $15 \mathrm{MHz}$ center frequency spacing. The third CC has $20 \mathrm{MHz}$ bandwidth with center frequency at $60 \mathrm{MHz}$

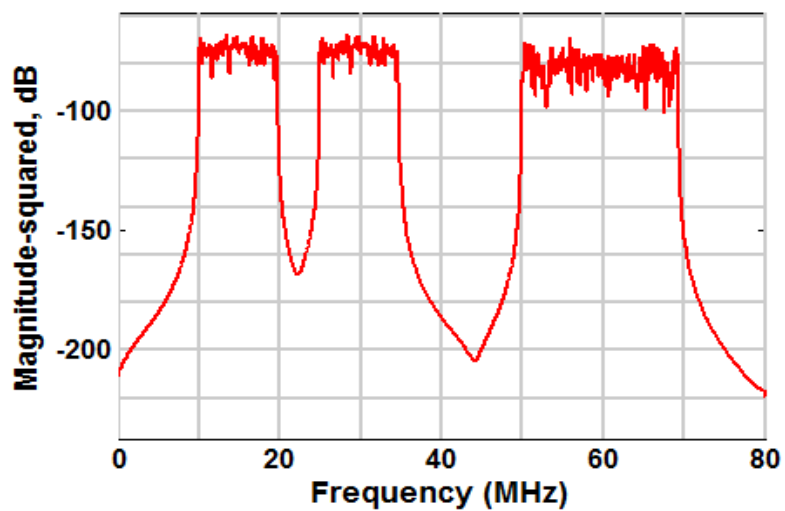

Fig. 18: Three CCs Non-Continuous Asymmetric CA $60 \mathrm{MHz}$ Bandwidth.

\subsubsection{Scenario D}

Fig. 19 shows the $100 \mathrm{MHz}$ CA LTE-A spectrum bandwidth with four CCs, each one has $20 \mathrm{MHz}$ bandwidth. The center frequency for the first $\mathrm{CC}$ is at $20 \mathrm{MHz}$, the second $\mathrm{CC}$ at $47 \mathrm{MHz}$, the third $\mathrm{CC}$ at $74 \mathrm{MHz}$ and the fourth $\mathrm{CC}$ at $101 \mathrm{MHz}$.

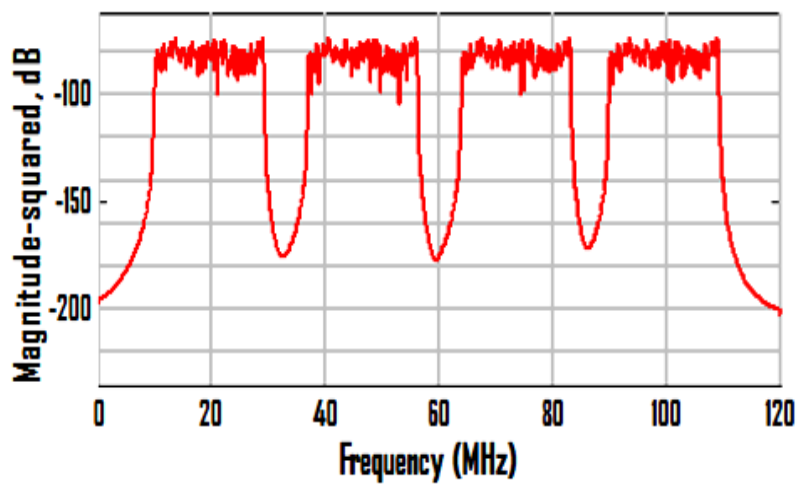

Fig. 19: Non-Continuous Symmetric CA $100 \mathrm{MHz}$ Bandwidth.

\subsubsection{Scenario E}

Fig.20 shows the $100 \mathrm{MHz}$ CA LTE-A spectrum bandwidth with asymmetric five CCs, four of them have $20 \mathrm{MHz}$ bandwidth and the last one has $10 \mathrm{MHz}$ bandwidth. The center frequency for the first $\mathrm{CC}$ is at $20 \mathrm{MHz}$, the second $\mathrm{CC}$ at $42 \mathrm{MHz}$, the third CC at $63 \mathrm{MHz}$, the fourth $\mathrm{CC}$ at $87 \mathrm{MHz}$ and the last $\mathrm{CC}$ at $105 \mathrm{MHz}$. The center frequency spacing between CC's satisfies the required multiple of $300 \mathrm{KHz}$.

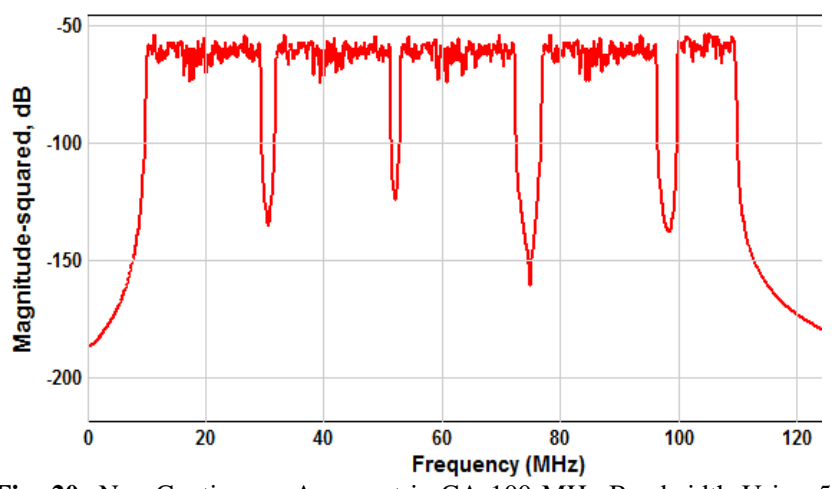

Fig. 20: Non-Continuous Asymmetric CA $100 \mathrm{MHz}$ Bandwidth Using 5 CCs.

So, the simulation results achieved the LTE-A requirements by using five CCs that expanded LTE band to $100 \mathrm{MHz}$.

\section{Conclusion}

This paper gives a description of LTE-A downlink physical layer. It contains a complete description of LTE-A block diagram consisting of channel coding, mapping and OFDM generator with the basic 3GPP OFDM parameter. The paper emphasizes the LTE-A $\mathrm{CA}$ with its different types. Therefore, different CA scenarios are implemented and tested. The first scenario is $30 \mathrm{MHz}$ symmetric CA using two CCs. The second scenario non-continuous Asymmetric CA $60 \mathrm{MHz}$ using two CCs. The third scenario is noncontinuous Asymmetric CA 60MHz bandwidth using three CCs. The fourth scenario is non-continuous symmetric CA $100 \mathrm{MHz}$ by using four CCs. The fifth scenario is non-continuous Asymmetric CA $100 \mathrm{MHz}$ by using five CCs that achieve the LTE-A requirement of band expansion with maximum number of CCs. This Simulink implementation can be considered as a first step for the software defined radio implementation of the LTE-A transceiver system. Such Simulink model can be converted into a digital signal processor code or VHDL code.

\section{References}

[1] 3GPP TR 36.913, Requirements for further advancements for Evolved Universal Terrestrial Radio Access (E-UTRA) (LTEAdvanced). Technical Report, European Telecommunications Standards Institute, 2010, 1-16.

[2] Jeanette Wannstrom, for 3GPP, LTE-Advanced. june 2103. www.3gpp.org (accessed Novamber 2016).

[3] Amitava Ghosh, Rapeepat Ratasuk, Bishwarup Mondal, Nitin Mangalvedhe, and Tim Thomas, Motorola Inc. "LTE-Advanced Next-Generation Wireless Broadband Technology." IEEE Wireless Communications, June 2010: 10-22. https://doi.org/10.1109/MWC.2010.5490974.

[4] Ian F. Akyildiz, David M. Gutierrez-Estevez, Elias Chavarria Reyes "The evolution to 4G cellular systems: LTE-Advanced." Physical Communication (Elsevier), 2010: 1-28.

[5] M.A.Mohamed, H.M.Abd-Elatty, M.E.A.Aboel-Seoud and W.M.Raslan. "Performance Analysis of LTE-Advanced Physical Layer." IJCSI International Journal of Computer Science Issues 11, No. 1 (2014): 80-87.

[6] Diksha Dugga, Jyoteesh Malhotra and Khushboo Arora."Performance Evaluation of Downlink Non Contiguous Carrier Aggregation in LTE-A." International Journal of Signal Processing, 
Image Processing and Pattern Recognition (ECE Department, Guru Nanak Dev University, RC Jalandhar) 8, no. 9 (2015): 275-282.

[7] Johansson, Tomi. 3GPP LTE Release 9 and 10 requirement and analysis to physical layer UE testing. UNIVERSITY OF HELSINKI Department of Computer Science, 2013.

[8] Kottkamp, Meik. LTE-Advanced Technology Introduction. White paper, Rohde \& Schwarz GmbH \& Co. KG, 2010, 1-22

[9] Abd-Elhamid M. Taha, Hossam S. Hassanein and Najah Abu Ali. LTE, LTE-Advanced and Wimax towards IMT-Advanced Networks. United Kingdom: John Wiley \& Sons, Ltd, 2012.

[10] Nokia, Networks. LTE-Advanced Carrier Aggregation Optimization. White Paper, Nokia, 2015, 1-16.

[11] Miller, Lirry. Carrier Aggregation Fundamentals for Dummies. New Jersey: John Wiley \& Sons, Inc, 2016.

[12] Carrier Aggregation in LTE-Advanced Nidhi Arora Asst Consultant, TCS Technical White Paper V1.0 March, 2013

[13] Technologist, Agilent. "CHAPTER 8 Looking Towards 4G: LTEAdvanced." In LTE and the Evolution to 4G Wireless: Design and Measurement Challenges, by Moray Rumney, Lead Technologist edited by Mory Rumney, 572-591. USA: Agilent Technologies,, 2014.

[14] Sklar, Bernard. Digital Communications Fundamentals and Applications. Second. California: Communications Engineering Services, Tarzana, California and University Of California, Los Angeles, 2001.

[15] Prof. Dr. Eng. Monica Borda. Fundamentals in Information Theory and Coding. Verlag Berlin Heidelberg: Springer, 2011.

[16] Lohith Kumar H G, Manjunatha K N, Suma M S, C K Raju, Prof.Cyril Prasanna Raj P. "Design and Performance Analysis of a 3GPP LTE/LTE-Advance Turbo Decoder Using Software Reference Models." International Journal of Scientific \& Engineering Research 2, No. 7 (July 2011): 1-4.

[17] Mr. Arvindraj Desai, Dr.M Baharthi, Mr.Nandeesh G M. "Carrier Aggregationin LTE-A for High Throughput." International Journal of Advanced Scientific and Technical Research 4, No. 4 (JulyAugust 2014): 659-667.

[18] Marwa Abdelfatah Abdeltwab, K. E. (2015)."Performance Improvement of OFDM Communication System in AWGN Channel". International Journal of Computer Applications, 110 (11), 17-23. https://doi.org/10.5120/19361-0969.

[19] Erik Dahlman, Stefan Parkvall, Johan Sköld and Per Beming. 3G Evolution: HSPA and LTE for Mobile Broadband. Edited By First. Uk: Elsevier Ltd, 2007.

[20] Tark Nasr Halawa, Ramy Ahmed Fathy,Abdelhalim Zekry. "Performance Analysis of LTE-A with 256-QAM." International Conference on Digital Information Processing Communication.2016.141145.https://www.researchgate.net/publication/301748 508_Performance_analysis_of_LTE-A_with_256-QAM

[21] Instruments, National. "Introduction to LTE Device Testing from Theory to Transmitter and Receiver Measurements." www.ni.com. January 22, 2015. www.ni.com/rf-academy (Accessed May 15, 2016).

[22] SONG, XUAN GUO \& PENGTAO. Simulink Based LTE System Simulator. Technical report, Department of Signals and Systems, Sweden: Chalmers University of Technology, 2010.

[23] Intini, Aníbal Luis. Orthogonal Frequency Division Multiplexing For Wireless Networks. University Of California Electrical and Computer Engineering Department, 2000.

[24] Pagès, Albert Serra. A Long Term Evolution Link Level Simulator. Master Thesis, Universitat Politècnica de Catalunya, 2009.

[25] Shahzad A. Malik, Madad Ali Shah,Amir H. Dar,Jahanzaib,Qamar Shahzad,Muhammad Saqib,Shahid A. Khan. "Evaluation of the Impact of Higher Order Modulation and MIMO for LTE Downlink." Australian Journal of Basic and Applied Sciences (Insinet), 2010: 4499-4508.

[26] Alaa Deshar Farhood, Neelesh Agarwal,A.K. Jaiswal, Navendu Nitin and Maham Kamil Naji. "Performance Analysis of OFDMA in LTE." International Journal of Current Engineering and Technology (INPRESSCO) 4, no. 3 (June 2014): 1614-1619.

[27] Nobuiko Mikio, Mikio Iwamura, Yoshihisa Kishiyama,Umesh Anil "CA for Bandwidth Extension in LTE-Advanced." NTT Docomo Technical Journal 12, No. 2 (2011): 10-19.

[28] 4G Americas. "LTE Carrier Aggregation." www.4gAmericas.org. October 1, 2014. (Accessed June 22, 2015).

[29] Jadon, Ekta Gujral and Jitendra Singh. "LTE Evolution towards Carrier Aggregation (LTE-Advanced)." Journal of Telecommunications System \& Management 5, No. 1 (2016): 1-5.

[30] Guangxiang Yuan, Xiang Zhang and Wenbo Wang. "Carrier Aggregation for LTE-Advanced Mobile Communication Systems."
IEEE Communications Magazine, February 2010: 88-93. https://doi.org/10.1109/MCOM.2010.5402669.

[31] Aws Zuheer Yonis and Mohammad Faiz Liew Abdullah. "Wider Bandwidth of non-Contiguous Component Carriers in LTEAdvanced." International Journal of Future Generation Communication and Networking 6, no. 2 (April 213): 49-62. 